\title{
A climate stress test of Los Angeles' water quality plans
}

\author{
Abdul Tariq ${ }^{1} \cdot$ Robert Jay Lempert $^{1} \cdot$ John Riverson $^{2}$ • \\ Marla Schwartz ${ }^{3} \cdot$ Neil Berg $^{1}$
}

Received: 31 October 2016 / Accepted: 18 August 2017 / Published online: 7 September 2017

(C) The Author(s) 2017. This article is an open access publication

\begin{abstract}
Climate change can significantly affect water quality, in addition contributing non-stationarity and deep uncertainty that complicates water quality management. But most of the total maximum daily load (TMDL) implementation plans crafted to meet water quality standards in the USA are developed assuming stationary climate and at best a small number of land use futures, although neither assumption seems reliably justified. To address this challenge, this study employs robust decision making (RDM) methods, commonly used to help develop water supply plans, to stress test the proposed Enhanced Watershed Management Plan (EWMP), a TMDL implementation plan, for the Tujunga Wash, the largest subwatershed of the Los Angeles River, over a wide range of climate and land use futures. We find that climate change could significantly reduce the ability of the Tujunga EWMP to meet water quality goals; however, meeting the city's goals for increasing permeable land cover offsets the risk of non-compliance in the face of climate change uncertainties. This study also introduces innovations in RDM analyses, including: treatment of the deeply uncertain incidence of extreme precipitation events, an explicit link between RDM scenario discovery methods and the specification of signposts for adaptive policy pathways, and the use of (imprecise) probabilistic climate projections to inform the choice among robust adaptive policy pathways. The paper also contributes to a larger debate over how to address climate and other uncertainties in regulatory processes involving water quality.
\end{abstract}

Electronic supplementary material The online version of this article (https://doi.org/10.1007/s10584-0172062-5) contains supplementary material, which is available to authorized users.

Robert Jay Lempert lempert@rand.org

1 RAND, Santa Monica, CA, USA

2 Paradigm, Fairfax, VA, USA

3 UCLA, Los Angeles, CA, USA 


\section{Introduction}

Climate change can significantly affect water quality (EPA 2010; Johnson et al. 2012; Fant et al. 2017), in addition contributing non-stationarity and deep uncertainty that complicates water quality management. For instance, many US jurisdictions develop total maximum daily load (TMDL) implementation plans that specify actions needed to meet federal and state water quality standards. But most TMDL plans are developed assuming stationary climate and at best a small number of land use futures, although neither assumption seems reliably justified (Fischbach et al. 2015).

To address this challenge, this study demonstrates the use of robust decision making $(\mathrm{RDM})$ as a means to evaluate and improve TMDL implementation plans. As described in more detail below, RDM provides a systematic, quantitative decision support methodology for developing robust and flexible plans under conditions of deep uncertainty (Lempert et al. 2003; Lempert and Collins 2007; Hallegatte et al. 2012). RDM has been used extensively for water supply management (Bureau of Reclamation 2012; Groves et al. 2014a, b). This study extends RDM to water quality by demonstrating a climate stress test of the Upper Los Angeles River Enhanced Watershed Management Plan (EWMP), a new TMDL implementation plan for the Tujunga Assessment Area (henceforth Tujunga EWMP). The Tujunga Wash is the largest subwatershed of the Los Angeles River.

This study introduces several innovations to the use of RDM for water quality management: treatment of the deeply uncertain incidence of extreme precipitation events, an explicit link between RDM scenario discovery methods (Lempert 2013) and the specification of signposts for adaptive policy pathways (Haasnoot et al. 2013; Kwakkel et al. 2016), and the use of (imprecise) probabilistic climate projections to inform the choice among robust adaptive policy pathways. This study builds on two previous case studies (Fischbach et al. 2015), which provided a first application of RDM to climate and water quality but did not fully engage with these topics.

The paper also contributes to a larger debate over how to address climate and other uncertainties in regulatory processes involving water quality. As part of their responsibilities under the Clean Water Act, jurisdictions are required to prepare TMDL implementation plans that specify the steps they will take to reduce the pollutants reaching impaired water bodies. The law requires that jurisdictions provide a reasonable assurance analysis (RAA) to demonstrate that the best management practices (BMPs) and other policy interventions proposed in the TMDL implementation plan will result in the required pollutant load reduction (RWQCB 2014). Jurisdictions often provide this RAA using rainfall runoff simulation models, informed by assumptions about future climate, hydrological, socio-economic, land use, and other conditions.

These analyses, and the regulatory processes in which they are embedded, do acknowledge uncertainty, for instance by including a margin of safety in projected load reduction (NRC 2000; Hantush 2009). More broadly, the US Environmental Protection Agency (EPA) explicitly uses an iterative risk management framework (Jones et al. 2014) to structure its engagement with local and regional partners in the development of TMDL implementation plans (EPA 1994). Many plans also include an adaptive management component designed to adjust a TMDL plan's BMPs and other interventions in response to new information. Such adaptive management is appropriate, because when faced with deep uncertainty, the best approach often seeks strategies that are robust over a wide range of futures and achieves that robustness through an adaptive process of acting, monitoring and potentially changing course in response 
to evolving conditions (Rosenhead 2001; Lempert et al. 2003; Walker et al. 2010). To date, however, even jurisdictions with access to sophisticated analytic capabilities have not been able to include such concepts, or even any significant representation of uncertainty, in their quantitative RAA, due in large part to the deep uncertainties involved and the difficulty of implementing many risk management frameworks with the highly complicated, detailed simulation models demanded by RAA. This study demonstrates how the simulation models currently used for water quality RAA, when employed with RDM, can support the development of robust adaptive TMDL plans under conditions of deep uncertainty.

The next section provides background on the current TMDL planning efforts on the Los Angeles River, Sect. 3 describes our approach, and Sect. 4 provides the results. The concluding section offers observations on the study's limitations and how RDM methods might improve the use of deeply uncertain climate information and the ability to conduct adaptive management for water quality.

\section{Background}

The Los Angeles Regional Water Quality Control Board, a state agency, has established various TMDLs in the Los Angeles River and tributaries for trash, nutrients, algae, toxics, salts, and metals (e.g., copper, lead, and zinc). ${ }^{1}$ To facilitate effective integrated water resource management, the Regional Board has issued a municipal separate storm sewer system (MS4) Permit to Los Angeles County, which allows county and city agencies flexibility to collaborate in the development and implementation of watershed management programs, including meeting the TMDLs. These agencies are currently in the final stages of developing TMDL implementation plans for review by the regional board.

To explore the impacts of climate change and other uncertainties on such TMDL implementation plans, this study focuses on the Tujunga Wash, the north-bank tributary of the Los Angeles River. The steep terrain of the Los Angeles National Forest comprises about three quarters of the Wash (165 mile ${ }^{2}$, upstream from 60 mile $^{2}$ of urbanized valley floor, home to over half a million residents. This subwatershed provides a good focus for this study because it faces significant climate uncertainty and is representative of both the orographic variability across the greater Los Angeles region and the jurisdictional complexity of the TMDL planning process.

The Los Angeles Bureau of Sanitation (LASAN), part of the City of Los Angeles Department of Public Works, leads the Upper Los Angeles River EWMP effort, which also includes Los Angeles County Flood Control District (LACFCD) and 14 other cities. These jurisdictions have developed a draft EWMP that includes the Tujunga Wash, described in Sect. 3 below, consisting of a portfolio of control measures optimized in the Tujunga Wash RAA to most cost-effectively meet the TMDL. This RAA currently assumes climate and land use remain stationary.

In recognition of uncertainty, the MS4 permit also calls for an adaptive management process, in which the permitee updates and revises the EWMP every 2 years based on observed progress towards achieving goals, achievement of interim milestones, and new information gained by the monitoring program, which might suggest improvements to RAA models and/or revisions of the expected performance of the BMPs.

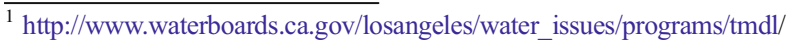




\section{Approach}

This study uses RDM to examine the uncertain impacts of climate change on the Tujunga Wash EWMP. As one of a class of "backwards" or "bottom up" approaches (Lempert et al. 2003; Kalra et al. 2014; Herman et al. 2015), RDM begins with a proposed strategy, stress tests it over a wide range of plausible futures, identifies scenarios that illuminate the vulnerabilities of the strategies, and uses this information to help identify and assess responses to reduce those vulnerabilities. This study implements RDM analysis by running the EWMP RAA simulation models to stress test the Tujunga EWMP over a wide range of future climate and land use conditions. The analysis then identifies a scenario that illuminates the vulnerabilities of the EWMP, uses this information to identify potentially more robust strategies that take the form of adaptive pathways, and estimates exceedance probabilities for the scenario that can inform tradeoffs among these strategies.

Like many RDM exercises, this project begins with a decision structuring exercise that organized the factors of analysis into an "XLRM" framework (Lempert et al. 2003). The letters $\mathrm{X}, \mathrm{L}, \mathrm{R}$, and $\mathrm{M}$ refer to: performance metrics (M) that reflect decision makers' goals; policy levers (L) that decision makers use to pursue their goals; uncertainties $(\mathrm{X})$ that may affect the connection between policy choices and outcomes; and relationships (R), often instantiated in simulation models, between outcomes to uncertainties and levers. This study's factors were developed in consultation with LASAN, LACFCD, and other stakeholders in the region. In particular, this study focuses on the zinc TMDL, which the RAA analysis identified as the limiting pollutant, and makes calculations for one time period, chosen at mid-century (20552065). This section briefly surveys these factors, with additional detail in the online supplement.

\subsection{Policy levers (L)}

As part of LASAN's planning process, the BMPs that comprise the Tujunga EWMP were optimized to most cost-effectively achieve the TMDL targets (CH2MHill and Paradigm 2015). LASAN generated a large number of BMP options and then used the RAA models to choose the most cost-effective portfolio assuming historical climate and current land use. The resulting Tujunga Wash EWMP, described in more detail in supplement, includes three categories of BMPs - regional projects (e.g., large, publically owned spreading basins), green streets (e.g., refurbishing city streets to increase permeability), and low impact development (e.g., revising building codes to increase storm water retention on private land) - which account for 45,22 , and 33\%, respectively, of the relative capacity needed to meet the zinc TMDL (CH2MHill and Paradigm 2015). The Tujunga EWMP is estimated to involve capital costs of $\$ 600$ million and $\$ 16$ million in annual operations and management costs.

As is common with RDM, this study uses this proposed Tujunga Wash EWMP as its starting point for its analysis of vulnerabilities and robust responses.

In addition to stress testing the current Tujunga EWMP, this study also considers alternative, potentially more robust, policies. While future land use is initially considered an uncertainty, Sect. 4 also estimates the additional BMPs that might be required to offset impacts of various future climates, as well as suggests how the EWMP might be configured as an adaptive strategy with signposts to monitor and contingency actions to take in response as future conditions unfold. 


\subsection{Uncertainties (X)}

This study considers future climate change and land use as uncertainties affecting the ability of the Tujunga EWMP to meet the zinc TMDL.

This study uses an ensemble of 47 climate projections prepared for LACFCD and the US Bureau of Reclamation for their LA Basin Study, which considered projections from three sets of publicly available models (Sankovich et al. 2013): 134 Coupled Model Intercomparison Phase 5 (CMIP5) projections downscaled using the Bias Corrected Constructed Analogues (BCCA) method, 112 Coupled Model Intercomparison Phase 3 (CMIP3) projections downscaled using the Bias Corrected Spatial Disaggregation (BCSD) method, and 100 CMIP5 projections downscaled using BCSD. All projections were downscaled to $1 / 8^{\circ} \times 1 / 8^{\circ}$ $(\sim 14 \mathrm{~km} \times 14 \mathrm{~km})$ spatial resolution.

As described in the supplement, the Basin Study chose a subset of 47 projections to sample extreme climate futures in Los Angeles. Out of the 134 CMIP5-BCCA projections, the LA Basin Study chose 16 projections from the low emissions trajectory (Representative Concentration Pathway 2.6), and 21 projections from the high emissions trajectory (RCP8.5), for a total of 37 CMIP5-BCCA projections. To select from among CMIP3-BCSD and CMIP5BCSD projections, LACFCD first considered five "climate scenarios" designated as 90th/90th (hot-wet), 90th/10th (warm-wet), 50th/50th (central tendency), 10th/10th (warm-dry), and 10th/90th (hot-dry) of temperature/precipitation percentiles, respectively, using the distribution of the suite of CMIP3-BCSD and CMIP5-BCSD projections. For each scenario, two separate climate time-series (one for CMIP3-BCSD and one for CMIP5-BCSD) was developed by blending ten projections in the underlying set that were closest to the temperature/precipitation quantiles of a given climate scenario (Sankovich et al. 2013). This resulted in five CMIP3$\mathrm{BCSD}$ projections and five CMIP5-BCSD projections representing a range of different emission scenarios.

In total, these 47 climate projections affect the Tujunga EWMP by changing the frequency and size of extreme precipitation events in the basin.

Our analysis also considers six future land use conditions reflecting a range of increasing intensity of low-impact development (LID) deployment in the region. The Baseline and Future SCAG futures represent estimates currently in the literature. LASAN used the former, which assumes current land use patterns will remain constant through 2050, to develop the Tujunga EWMP. Our Future SCAG future uses the 2035 land use projection from the Southern California Association of Governments (SCAG 2015a, b). In addition, this study crafted four additional land use futures with increasingly aggressive LID adoption, using estimates by a subcommittee of the LA Basin Study Stakeholder Technical Advisory Committee (STAC) (Alexanderson and Bradbury 2013). This study's Moderate LID and Improved LID are both based on the Future SCAG but with enhanced permeability for each parcel of 10 and 20\%, respectively. To reflect the City of Los Angeles' recent Low Impact Development Ordinance (LASAN 2011), we crafted an Ordinance plus future, which assumes that all new development in the Tujunga Wash is fully permeable. Finally, the Most Optimistic future begins with Ordinance plus, and additionally assumes that the permeability of each parcel of existing development is increased by the most optimistic estimates made by the STAC.

Overall, these alternative land use futures affected total impervious cover that ranged from about one third to one half of the total area of Tujunga Wash. The impervious cover affects the Tujunga EWMP by changing the amount of runoff from any given precipitation event. 


\subsection{Relationships (R)}

To stress test the Tujunga EWMP, we use LACFCD's Watershed Modeling and Management System (WMMS) simulation model. The WMMS platform is a comprehensive model of the Los Angeles watershed, developed and calibrated to adequately represent the hydrology and water quality in the region (LACDPW 2010a, b; ULAR 2016). We use two WMMS modeling tools: (1) EPA's Loading Simulation Program in C++ (LSPC) calibrated for the Los Angeles River watershed and used to generate land use runoff and pollutant loads and (2) EPA's System for Urban Stormwater Analysis and INtegration (SUSTAIN) which models zinc load reductions from EWMP BMPs. As described in the supplement, the LSPC model was calibrated to historic rainfall. The base case reference point for comparing all futures is the effluent zinc load for historical climate and historical land use projections, assessed downstream of EWMP BMPs.

LSPC was used to generate hourly runoff and pollutant loadings for zinc for all climate futures for the years 2055-2065. For each future, we identified the 90th percentile 24-h runoff event. We focused on this event, because it is used to define the regulatory standard that the EWMP seeks to meet. Because the spatial resolution of the climate projections is coarse compared to the topography of the Tujunga Wash, we statistically derived a representative rainfall depth associated with the 90th percentile event by first area-weighting rainfall time series from the 21 rainfall gages upstream in Wash to find a normalizing depth and then proportionally scaling that depth by rainfall gage to retain the relative variability of rainfall. This process implicitly assumes that the spatial distribution of extreme precipitation events does not vary across the climate projections, an assumption that could be usefully examined in future work. For each climate and land use future, runoff from that event was routed through the optimized EWMP BMP network.

\subsection{Performance metrics (M)}

For each model run, we determine if the mid-century (2055-2065) critical condition zinc load exceeds the base case critical condition, defined as the 90th percentile zinc exceedance runoff load for the historical climate projection (1986-2012) with current land use assessed downstream of the Tujunga EWMP BMPs. As described below, we also estimate the change in 20year implementation costs of adding BMPs to the current Tujunga EWMP to mitigate against the increased zinc load from climate change.

\subsection{RDM process}

Using these XLRM factors, this study follows the subsequent steps of an RDM analysis: generating cases by stress testing the proposed strategy in a wide range of plausible futures, using the resulting database of model runs to identify scenarios that illuminate vulnerabilities of the strategy, identifying potentially more robust strategies based on the information in these scenarios, and assessing the resulting tradeoffs (Lempert 2013; see Fig. 1 and updated version in supplement).

Case generation This study stress tests the Tujunga EWMP by running 282 plausible futures (47 climate $\times 6$ land use futures) with the WMMS model, assessing results both upstream and downstream of the EWMP BMPs, for a total of 564 cases $(47 \times 6 \times 2)$. 


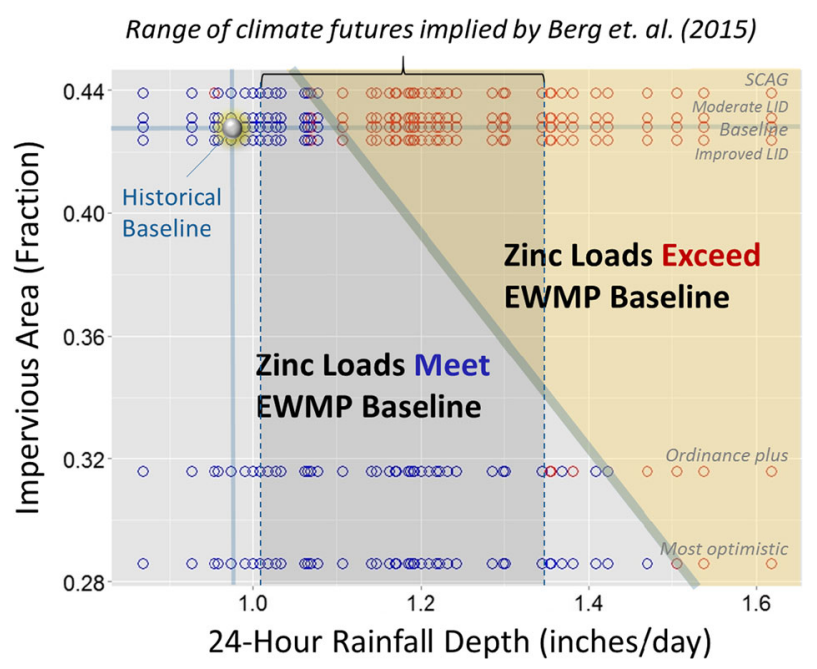

Fig. 1 Scatterplot of futures in which the Tujunga EWMP meets and exceeds zinc TMDL. Blue/red dots indicate futures in which the TMDL is met/exceeded. Gray dot indicates historic climate and current land use. The diagonal line marks the non-compliance scenario's border. Dashed vertical lines mark range of future 24-h precipitation derived from Berg et al. (2015)

Scenario that illuminate vulnerabilities The study uses a scenario discovery classification algorithm (Bryant and Lempert 2010) on the database of cases to identify factors that best distinguish the futures in which the Tujunga EWMP meets and misses its zinc TMDL. In particular, this study uses a simplified version of the approach described in Dalal et al. (2013), using both the principal component analysis and the PRIM (patient rule induction method) (Friedman and Fisher 1999) algorithms to identify the scenario that best illuminates the vulnerabilities of the Tujunga EWMP. The land use inputs are characterized by the percentage of impervious area. To characterize the climate time-series with a small number of parameters, we calculate 11 statistical summaries for each: average annual rainfall and its standard deviation, average annual potential evapotranspiration (PET) and its standard deviation, the 90th percentile of average annual rainfall, the 90th percentile of the average PET, the average rainfall in a 24-h period and its standard deviation, the average PET in a 24-h period and its standard deviation, and the 90th percentile area-weighted ${ }^{2}$ rainfall in a $24-\mathrm{h}$ period.

New options The scenario discovery analysis suggests that the Tujunga EWMP fails to meet its zinc TMDL in futures characterized by extreme precipitation events and a relatively impermeable urban land surface. The study identifies potential strategies that might reduce these vulnerabilities in two steps: (1) estimating the additional BMPs that would be needed to meet the zinc TMDL in this scenario and (2) suggesting contingent response, which might employ these additional BMPs, if certain early warning signs associated with the scenario were observed.

To obtain a simple estimate of additional BMPs required in the vulnerable scenario, we first estimate the additional load reduction required in each future contained in the scenario. We

$\overline{{ }^{2} \text { This is the 90th percentile rainfall }}$ depth for the area-weighted (over the 21 weather stations) Tujunga Wash average. 
then estimate the additional BMP capacity needed to provide this additional load reduction by combining results from our model runs with cost-effectiveness curves from the optimized BMP portfolio from the Tujunga EWMP (CH2MHill and Paradigm 2015). To calculate the additional load reduction required, we calculate the load reduction achieved with the Tujunga EWMPs for each land use future in the baseline climate future. This yields the theoretical maximum load reduction for each land use, assuming historic climate. We then calculated the load reduction achieved with EWMPs for the 90th percentile climate future (in terms of the amount of precipitation) and estimate the additional BMP capacity required in each land use scenario to meet the zinc TMDL for the 90th percentile climate future.

As described in the results section below, this simple, approximate approach suggests new adaptive options that appeared to provide useful information to LASAN. The approach does not yield fully dynamic adaptive pathways (Haasnoot et al. 2013) because conducting a full reoptimization of the BMPs for each future, or robust optimization over all the futures, was beyond the study's scope. The supplement describes a more detailed approach that might be employed in the future.

Assessing tradeoffs To assist decision makers in choosing between this adaptive pathway and the current Tujunga EWMP, the study provides a bounding set of likelihood estimates for the scenario that illuminates the EWMP's vulnerabilities. This analysis is also presented in Sect. 4.

\section{Results}

Table 1 reports the initial results of the stress test of the Tujunga EWMP. The table shows the percentage of mid-century climate futures that meet baseline zinc loads for each land use future, with and without the Tujunga EWMP BMPs (calculated downstream and upstream, respectively). Under Baseline land use, only one (2\%) of the climate future meet the baseline zinc loads assessed without BMPs. With the BMPs, about a third (33\%) of the futures meet baseline zinc loadings. Table 1 also highlights the importance of low-impact development in improving water quality. The number of futures that exceed zinc baseline with the Most Optimistic land use but without the EWMP is the same as Baseline land use with the Tujunga EWMP in place.

Table 1 Percent of climate futures meeting zinc TMDL under future land use conditions

\begin{tabular}{lll}
\hline Land use condition & \multicolumn{2}{c}{ Percent (\%) climate futures meeting Tujunga baseline zinc loads } \\
\cline { 2 - 3 } & Assessed upstream of EWMP BMPs & Assessed downstream of EWMP BMPs \\
\hline Baseline & $2 \%$ & $33 \%$ \\
SCAG & $8 \%$ & $29 \%$ \\
Moderate LID & $10 \%$ & $33 \%$ \\
Improved LID & $10 \%$ & $33 \%$ \\
Ordinance plus & $29 \%$ & $83 \%$ \\
Most optimistic & $33 \%$ & $92 \%$ \\
\hline
\end{tabular}

Note: These percentages refer to the number of model runs in our experimental design, not probabilities. Each model run represents one future, which is one mapping of assumptions to consequences, and is not assumed to be equally likely 
Figure 1 presents the results of the scenario discovery analysis on this data. Blue circles indicate futures that meet the zinc target, while red circles indicate exceedance. The figure's axes display the two factors identified by the scenario discovery analysis as most important in distinguishing the futures where the Tujunga EWMP meets and exceeds its zinc TMDL goal. These factors are the 90th percentile of 24-h precipitation (horizontal axis) and percent impervious area in the basin (vertical axis). The relationship

$$
\text { Imperviousness }(\%)+0.35^{*} 24-\text { hour Precipitation }\left(90^{\text {th }}\right)(\text { inches })>0.81
$$

defines the border of the scenario that illuminates the mid-century vulnerabilities of the Tujunga EWMP, as shown by the thick diagonal line in Fig. 1. Note that, in general, the effluent zinc load meets (exceeds) the Baseline in futures that lie to the lower left (above right) of this line. The Tujunga EWMP was developed for the Baseline land use future and the historical 90th percentile 24 -h critical condition, marked by the gray dot at $0.95 \mathrm{in}$., which is to the lower left of the scenario boundary. The boundary's linear combination of impervious area and extreme precipitation reflects the fact that urban runoff is the underlying factor driving the zinc loading in the Tujunga Wash. The intensity of urban runoff is a function of water volume, given by precipitation, and transport efficiency, given by impervious area.

\subsection{Adaptive management of the Tujunga EWMP}

The MS4 permit calls for an adaptive management process to manage uncertainty in the Tujunga Wash. Such a process might address the potential vulnerabilities of the EWMP shown in Fig. 1, and the information in the figure can help inform an appropriate adaptive management process (Groves et al. 2014a, b).

The first step is to estimate the additional BMPs required to meet the zinc TMDL in the vulnerable scenario. For Baseline land use, as shown in Fig. 2, meeting the TMDL requires roughly 230 additional acres-feet of storm water capture capability, consisting of about 77 additional acres-feet of Green Streets and 153 additional acres-feet of Regional Projects. This additional load reduction comes at an estimated additional 20-year implementation cost of $\$ 500$ million. In the Ordinance plus future, however, virtually no additional BMPs would be required.

We next use the information in Figs. 1 and 2 to describe two alternative adaptive pathways. Each pathway begins with a near-term plan for BMP deployments, signposts to monitor, and planned adjustments to the BMP deployments if the monitoring suggests that they are necessary (Lempert and Groves 2010).

The first pathway begins with the current Tujunga EWMP, as shown in Fig. 3. For signposts of land use/land cover, greenness and permeability of the land surface are related to features of the urban landscape that can be observed. For instance, Lee et al. (2017) combined aerial photography over Los Angeles County and official records of property characteristics to monitor land cover changes (notably grass and trees/shrubs) from 2000 to 2009 in singlefamily neighborhoods. For neighborhoods in the Tujunga Wash, green cover was reduced by over $25 \%$. By translating land cover changes to permeability changes (for instance, through published lookup tables), alongside additional remote-sensing measurements, one could provide a tractable monitoring of near-real time land use changes in urban settings.

Using this or similar techniques, LASAN can monitor land use trends and determine in the next decade or two which land use future will come to pass. The right panel in Fig. 3 shows the 


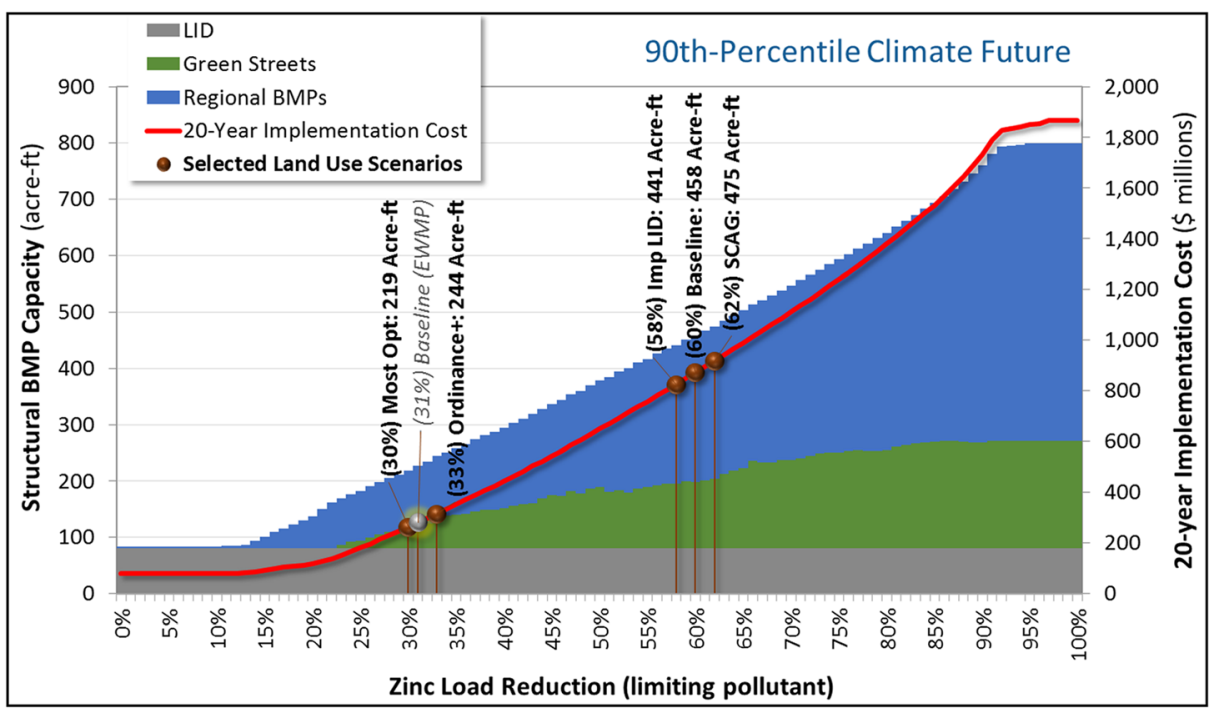

Fig. 2 Additional BMP capacity needed to meet zinc TMDL for various land use futures, showing the most cost-effective mix of BMPs for each load reduction, the associated 20-year implementation costs

additional BMPs required beyond those in the Tujunga EWMP to meet the zinc baseline in the 90th percentile future climate in mid-century as a function of the land use future. If land use monitoring suggests that one of the more intensive LID futures-Ordinance plus or Most Optimistic - are coming to pass, LASAN needs to make at most small adjustments to its BMP deployment. However, if land use monitoring suggests that one of the less-LID-intensive futures - such as Baseline - is coming to pass, the strategy would shift to a pathway with a larger deployment of Green Streets and a significantly larger deployment of Regional Projects.

The second pathway shown in Fig. 3 begins by planning for an augmented set of BMPs, appropriate for the Baseline future. If the land use monitoring suggests that one of the more intensive LID futures is coming to pass, the strategy would shift to the current BMP pathway by canceling the deployment of the additional BMPs. However, if the land use monitoring

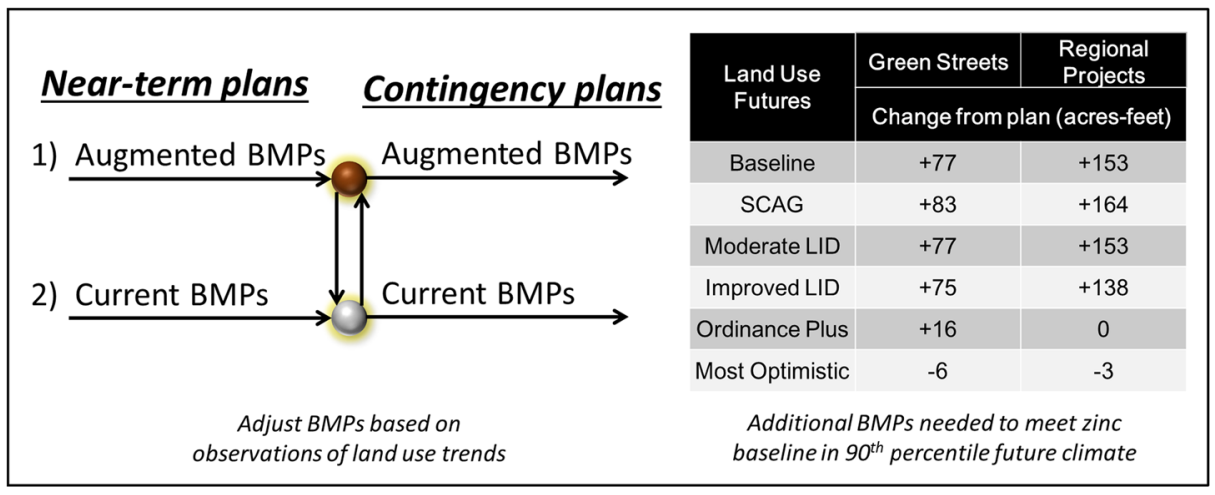

Fig. 3 Potential adaptive pathways: right panel shows additional BMPs required, beyond current EWMP, to meet zinc TMDL in the 90th percentile climate; left panel shows two pathways that meet the TMDL by monitoring future land use 
suggests that one of the less-LID-intensive futures - such as Baseline - is coming to pass, the strategy would remain on the augmented BMP pathway.

Note that neither of these options considers monitoring climate trends to determine whether the 90th percentile 24-h rain event in the Tujunga Wash is becoming more extreme. At present, no such climate signposts seem available. For example, the ensemble of CMIP5 climate projections used in this study show no temporal trend in extreme flow years in Los Angeles for the twenty-first century. This lack of any clear trend is consistent with a previous finding that downscaled climate models within the CMIP3 ensemble produce divergent projections on intense daily precipitation changes over California (Pierce et al. 2013). As discussed in the supplement, climate signposts might become possible in the future.

\subsection{Estimating the likelihood of the Tujunga EWMP's non-compliance scenario}

Should decision makers retain the current Tujunga EWMP or adopt one of the two alternative adaptive pathways? If the later, which one? As one important input to this choice, this study uses alternative sets of climate information to provide a bounding set of probability distributions for the future 90th percentile 24-h rainfall.

Berg et al. (2015) provide a best-estimate probability distribution for future climate change in the Los Angeles basin. This work used a combination of dynamical and statistical downscaling techniques to generate 2-km resolution projections of wintertime (DecemberMarch) mid-twenty-first century (2041-2060 average minus 1981-2000 average) precipitation changes over the greater Los Angeles region. Specifically, Berg et al. (2015) dynamically downscaled mid-century climate changes based on projections from 5 CMIP5 GCMs. Within this five-model ensemble, the authors identified a single high-resolution spatial pattern that largely captured wintertime precipitation changes across the region. By relating this spatial pattern to GCM-based precipitation changes through regression techniques, Berg et al. (2015) statistically downscale mid-century precipitation changes for 36 CMIP5 GCMs and all RCPs and thus generate a probability density function for future changes in average annual precipitation in the LA basin.

Applying this distribution to the ensemble of climate projections used in this study yields the range of 90th percentile 24-h rainfall marked by vertical dashed lines in Fig. 1. ${ }^{3}$ Note that for the Baseline land use future, most of this range lies within the non-compliance scenario, but for the Ordinance plus future, the non-compliance scenario lies entirely outside this range. The EWMP thus exceeds zinc TMDL in most climate futures with Baseline land use, but meets the TMDL in all likely climate futures with the Ordinance plus future. We can further quantify these statements by noting that only $37 \%$ of the climate futures within the Berg et al. (2015) range meet the zinc TMDL, suggesting that the probability of meeting the zinc TMDL with climate change is significantly less than $40 \%$ with Baseline land use ${ }^{4}$ and $100 \%$ for Ordinance plus. This result is consistent with the expectation that climate change will likely increase the intensity of extreme storms independent of any changes in annual average precipitation.

One obtains similar, but less optimistic, results assuming an equal weighting over all 47 climate projections considered in this study. This equal weighting assumption suggests a $33 \%$

\footnotetext{
${ }^{3}$ To derive the 90th percentile 24-h rainfall window implied by the Berg et al. (2015) data, we matched their 5th and 99th percentile average yearly rainfall to corresponding climate projections used in our analysis and used the 90th percentile 24-h rainfall for these projections as the lower and upper bounds shown in Fig. 1.

${ }^{4}$ The probability is likely significantly lower than $40 \%$ because the Berg et al. (2015) distribution is peaked to the right of the boundary of the vulnerable scenario.
} 
likelihood of meeting the zinc TMDL for the Baseline and $83 \%$ for the Ordinance plus future. While the Berg et al. (2015) range likely provides a better estimate, it is useful to note that even the CMIP3 and CMIP5 ensembles likely under-sample the extreme tails of the actual distribution of future climate. One reason for this is that the ensemble of best estimates of climate model parameters like climate sensitivity (as used in the CMIP analyses) does not sample the high tail of parameter estimates (compare, for example Bindoff et al. (2013) with Olson et al. 2012). As a result, an analysis based on CMIP ensembles may overestimate the ability of the Tujunga EWMP to meet the zinc TMDL.

These estimates suggest that even if decision makers were entirely confident in the Berg et al. (2015) distributions, the choice to rely entirely on the current Tujunga EWMP would imply an estimate that the likelihood of the Ordinance plus future was over $90 \% .^{5}$ Unless decision makers have reason for such confidence, they might adopt one of the adaptive pathways strategies. The choice between the two adaptive pathways might depend both on the degree of confidence in the Ordinance plus future and on the extent to which today's decision makers can demonstrate a future ability to expand the BMP deployment if necessary. For instance, the pathway that begins with the current Tujunga EWMP would also involve developing the capability to monitor land use trends and preparing for the future possibility of augmenting the BMPs, perhaps by identifying sites for future regional projects.

\section{Conclusions}

Government agencies charged with meeting regulatory water quality standards face a difficult challenge of planning under deep uncertainty. On the one hand, climate change and other uncertainties can create potential, hard-to-predict vulnerabilities for many water quality implementation plans. A plan expected to be cost-effective and compliant with a TMDL in one climate future may prove non-compliant and/or too costly if another comes to pass. Probabilistic forecasts of future climate, in particular, of the extreme events often most important to water quality, are currently unreliable. The best response to such uncertainty is often to adopt a robust and flexible plan, ones designed to adjust over time in response to new information in order to perform well (i.e., be robust) over a wide range of plausible futures. On the other hand, public agencies are expected to conduct an accountable, objective, and predictable planning process to demonstrate future compliance with regulatory mandates (Fischbach et al. 2015).

In principle, US law provides agencies broad leeway to develop flexible and robust water quality implementation plans. However, adaptive management plans have been rejected in court because they lack sufficient specificity on how they will achieve their regulatory mandates. For example, a US Court of Appeals recently noted (NRDC vs. Pritzker No. 1416375 ) that "just as it is not enough to simply invoke 'scientific uncertainty' to justify an agency action, it is not enough to invoke 'adaptive management' as an answer to scientific uncertainty." In addition, some stakeholder groups express distrust with adaptive management because they perceive it as a means to defer hard but uncertain decisions to the future without any means for current stakeholders to commit their successors to taking action in the future if needed.

This study demonstrates a systematic, straight-forward to implement, analytic framework for incorporating climate and other uncertainties into water quality implementation plans. This framework allows incorporation of deeply uncertain scientific information into the regulatory

${ }^{5}$ Assuming a desire for $95 \%$ reliability of compliance, $p \cdot 100 \%+(1-p) \cdot 40 \%>95 \%$ requires $p>92 \%$. 
planning, in particular, information that does not support high confidence estimates of probabilistic risk. Where appropriate, the framework supports a transparent, objective, and predictable process of structuring adaptive management plans. Developing adaptive management plans through such a process might help them survive legal challenge and provide sufficiently well-defined signposts and contingent response to enhance confidence that needed future actions would in fact be implemented. ${ }^{6}$

Applying the RDM approach to the Tujunga EWMP, this study finds that by mid-century, climate change could significantly reduce the ability of the plan to meet its zinc TMDL. The key drivers of the non-compliance scenario are the intensity of the 90th percentile 24-h rainfall and the percentage of effective impervious cover in the basin. If future land use resembles current land use, the Tujunga EWMP would meet the zinc TMDL in less than $40 \%$ of climate futures. If however, Los Angeles successfully implements its aggressive stormwater management ordinance, designed to significantly increase the city's permeable land cover, it will largely eliminate the EWMP's climate-change induced non-compliance risk.

Eliminating this non-compliance risk in Tujunga Wash with a safety margin approach could require roughly an additional 230 acres-feet of BMPs at a 20-year implementation cost of $\$ 500$ million, unless the city could guarantee that is aggressive permeability goals for land cover would be met.

Alternatively, to address the uncertainty affecting water quality in the Tujunga Wash, Los Angeles might adopt an adaptive management plan that begins with the current Tujunga EWMP's planned BMP deployment, monitors land use trends, and increases the BMP deployment if land use does not evolve as envisioned by the stormwater management ordinance.

This analysis suggests concrete questions that could inform legal and political scrutiny of such an adaptive management plan, for instance, the extent to which LASAN has the capability to monitor land use trends and the extent to which the agency is prepared to take the proposed contingency actions if they prove necessary.

This study has important limitations, such as unaddressed uncertainties that might prove relevant to TMDL implementation plans. These include the efficacy of various BMPs, in particular those involving green infrastructure; uncertainties in hydrologic flows that might be represented by alternative rainfall-runoff models; and uncertainty in the spatial distribution of extreme precipitation events. In addition, zinc may not remain the Tujunga Wash's limiting pollutant in the future, since a significant source, the brake pads of cars, might be affected by future changes automobile technology such as regenerative braking. In principle, the RDM approach could address such uncertainties, but they were beyond the scope of this study. As described in more detail in the supplement, this study suggests that the rainfall-runoff and BMP optimization simulation models currently used for TMDL planning are adequate for this type of RDM analysis but could be improved.

Overall, this study suggests that climate change and land use can significantly affect TMDL implementation plans; identifies how one such plan might be modified to address the resulting vulnerabilities; and demonstrates how robust decision making methods, employed with existing simulation models, may be able to generate legally acceptable plans that are robust and flexible in the face of climate and other uncertainties.

\footnotetext{
${ }^{6}$ The authors thank Edward Parson and Sean Hecht of the Emmett institute on Climate Change and the Environment at UCLA Law School for useful discussions on this topic.
} 
Acknowledgements The authors thank the US Environmental Protection Agency for its generous support of this work under contract EPG13C-00395. We also thank Shahram Kharaghani and Hubertus Cox of the Los Angeles Bureau of Sanitation, Lee Alexanderson and TJ Moon of the Los Angeles County Flood Control District, Edith de Guzman of TreePeople, and Susan Julius and Tom Johnson of US EPA for their advice and guidance throughout the course of this study.

Open Access This article is distributed under the terms of the Creative Commons Attribution 4.0 International License (http://creativecommons.org/licenses/by/4.0/), which permits unrestricted use, distribution, and reproduction in any medium, provided you give appropriate credit to the original author(s) and the source, provide a link to the Creative Commons license, and indicate if changes were made.

\section{References}

(NRC), N. R. C (2000) Assessing the TMDL approach to water quality management. Committee to assess the scientific basis of the total maximum daily load approach to water pollution reduction. National Research Council, Washington D.C.

Alexanderson L, Bradbury D (2013) Los Angeles basin stormwater conservation study, task 3.2. Hydrologic modeling report. County of Los Angeles Department of Public Works Los Angeles County Flood Control District, Los Angeles

Berg N, Hall A, Sun F, Capps S, Walton D, Langenbrunner B, Neelin D (2015) Twenty-first-century precipitation changes over the Los Angeles region. J Clim 28(2):401-421

Bindoff NL et al (2013) Detection and attribution of climate change: from global to regional. In: Stocker TF, Qin D, Plattner GK, Tignor M, Allen SK, Boschung J, Nauels A, Xia Y, Bex V, Midgley PM (eds) Climate change 2013: the physical science basis. Contribution of Working Group I to the fifth assessment report of the intergovernmental panel on climate change. Chapter 10. Cambridge University Press, Cambridge, pp $867-952$.

Bryant BP, Lempert RJ (2010) Thinking inside the box: a participatory, computer-assisted approach to scenario discovery. Technol Forecast Soc Chang 77:34-49

Bureau of Reclamation (2012) Colorado River Basin water supply and demand study: study report. United States Bureau of Reclamation, Washington D. C, p 89

CH2MHill and Paradigm (2015) Enhanced Watershed Management Program (EWMP) for the Upper Los Angeles River Watershed

Dalal S, Han B, Lempert R, Jaycocks A, Hackbarth A (2013) Improving scenario discovery using orthogonal rotations. Environ Model Softw 48:1-16

Environmental Protection Agency (EPA) (2010) National water program strategy: response to climate change key action update for 2010-2011. US Environmental Protection Agency

Fant C, Srinivasan R, Boehlert B, Rennels L, Chapra SC, Strzepek KM, Corona J, Allen A, Martinich J (2017) Climate change impacts on US water quality using two models: HAWQS and US basins. Water 9:118

Fischbach JR, Lempert RJ, Molina-Perez E, Tariq A, Finucane ML, Hoss F (2015) Managing water quality in the face of uncertainty: a robust decision making demonstration for EPA's National Water Program. RAND, Santa Monica

Friedman JH, Fisher NI (1999) Bump hunting in high-dimensional data. Stat Comput 9:123-143

Garner G, Keller K (submitted) When the tail wags the decision

Groves DG, Bloom EW, Lempert RJ, Fischbach JR, Nevills J, Goshi B (2014a) Developing key indicators for adaptive water planning. J Water Resour Plan Manag. https://doi.org/10.1061/(ASCE)WR.19435452.0000471 05014008-05014001-05014008-05014010

Groves DG, Fischbach JR, Kalra N, Molina-Perez E, Yates D, Purkey D, Fenci A, Mehta VK, Wright B, Pyke G (2014b) Developing robust strategies for climate change and other risks: a water utility framework. Water Research Foundation, New York State Energy \& Development Authority (NYSERDA) and Water Services Association of Australia (WSAA), Denver

Haasnoot M, Kwakkel JH, Walker WE, ter Maat J (2013) Dynamic adaptive policy pathways: a new method for crafting robust decisions for a deeply uncertain world. Glob Environ Chang 23(2):485-498

Hallegatte S, Shah A, Lempert R, Brown C, Gill S (2012) Investment decision making under deep uncertainty: application to climate change. Washington, DC, World Bank

Hantush MM (2009) Estimation of TMDLs and margin of safety under conditions of uncertainty. World Environmental \& Water Resources Congress, Kansas, MO, May 17-21, 2009. American Society of Civil Engineers (ASCE), Reston 
Herman J, Reed P, Zeff H, Charackiis G (2015) How should robustness be defined for water systems planning under change? J Water Resour Plan Manag. https://doi.org/10.1061/(ASCE)WR.1943-5452.0000509

Johnson TE, Butcher JB, Parker J, Weaver CP (2012) Investigating the sensitivity of U.S. streamflow and water quality to climate change: U.S. EPA Global Change Research Program's 20 Watersheds Project. J Water Resour Plan Manag 138(5):453-464

Jones RN, Patwardhan A, Cohen S, Dessai S, Lammel A, Lempert R, Mirza MMQ, Storch HV (2014) Chapter 2. Foundations for decision making. Climate Change 2014: impacts, adaptation, and vulnerability. Intergovernmental Panel on Climate Change (IPCC)

Kalra N, Hallegatte S, Lempert R, Brown C, Fozzard A, Gill S, Shah A (2014) Agreeing on robust decisions: a new process of decision making under deep uncertainty. Policy research working paper. W. Bank, World Bank

Kwakkel JH, Haasnoot M, Walker WE (2016) Comparing robust decision-making and dynamic adaptive policy pathways for model-based decision support under deep uncertainty. Environ Model Softw 86:168-183

LACDPW (2010a) Los Angeles County watershed model configuration and calibration-part I: hydrology. Prepared for County of Los Angeles Department of Public Works, Watershed Management Division. Tetra Tech, Los Angeles County

LACDPW (2010b) Los Angeles County watershed model configuration and calibration - part II: water quality. Prepared for County of Los Angeles Department of Public Works, Watershed Management Division. Tetra Tech, Los Angeles County

LASAN (2011) Storm water ordinance. Ordinance No. 181899. C. o. L. A. B. o. Sanitation

Lee SJ, Travis L, Rich C, Wilson JP (2017) Increased home size and hardscape decreases urban forest cover in Los Angeles County's single-family residential neighborhoods. Urban Forestry \& Urban Greening 24:222235

Lempert R (2013) Scenarios that illuminate vulnerabilities and robust responses. Clim Chang 117:627-646

Lempert RJ, Collins M (2007) Managing the risk of uncertain threshold responses: comparison of robust, optimum, and precautionary approaches. Risk Anal 27(4):1009-1026

Lempert R, Groves DG (2010) Identifying and evaluating robust adaptive policy responses to climate change for water management agencies in the American West. Technol Forecast Soc Chang 77:960-974

Lempert RJ, Popper SW, Bankes SC (2003) Shaping the next one hundred years: new methods for quantitative, long-term policy analysis. RAND Corporation, Santa Monica

Olson R, Sriver R, Goes M, Urban NM, Matthews HD, Haran M, Keller K (2012) A climate sensitivity estimate using Bayesian fusion of instrumental observations and an Earth System model. J Geophys Res: Atmos 117(D4). https://doi.org/10.1029/2011JD016620

Pierce DW, Cayan DR, Das T, Maurer EP, Miller NL, Bao Y, Kanamitsu M, Yoshimura K, Snyder MA, Sloan LC, Franco G, Tyree M (2013) The key role of heavy precipitation events in climate model disagreements of future annual precipitation changes in California. J Clim 26:5879-5896

Rosenhead J (2001) Robust analysis: keeping your options open. In: Rosenhead J, Mingers J (eds) Rational analysis for a problematic world revisited: problem structuring methods for complexity, uncertainty, and conflict. Wiley, Chichester

RWQCB (2014) Guidelines for conducting reasonable assurance analysis in a watershed management program, including an enhanced watershed management program. Los Angeles Region Water Quality Control Board, Los Angeles

Sankovich V, Gangopadhyay S, Pruitt T, Caldwell RJ (2013) Los Angeles basin stormwater conservation study task 3.1. Development of climate-adjusted hydrologic model inputs, US Bureau of Reclamation

SCAG (2015a) 2012 existing land use data. Southern California Association of Governments

SCAG (2015b) 2035 general plan land use projection data. Southern California Association of Governments

ULAR (2016) Enhanced Watershed Management Program (EWMP) for the Upper Los Angeles River Watershed. U. L. A. R. W. M. Group, Black \& Veatch Team. Preparation Leads: CH2M and Paradigm Environmental

U.S. Environmental Protection Agencgy (EPA) (1994) Water quality standards handbook. U.S. Environmental Protection Agency, Washington, D.C.

Walker W, Marchau V, Swanson D (2010) Addressing deep uncertainty using adaptive policies. Technol Forecast Soc Chang 77:917-923 